\title{
Identification of Differentially Expressed Genes by cDNA-AFLP in Magnaporthe oryzae
}

\author{
*Corresponding author \\ Tel: +82-61-750-3868 \\ Fax: +82-61-750-3868 \\ E-mail: spark@scnu.ac.kr \\ ORCID \\ https://orcid.org/0000-0003-1267-1111
}

Received November 24, 2019

Revised December 10, 2019

Accepted December 10, 2019

\author{
Myoung-Hwan $\mathrm{Chi}^{1}$ and Sook-Young Park ${ }^{2 *^{*}}$ \\ ${ }^{1}$ Plant Biology Division, Noble Research Institute, Ardmore, OK 73401, USA \\ ${ }^{2}$ Department of Plant Medicine, Sunchon National University, Suncheon 57922, Korea
}

\begin{abstract}
Analysis of differentially expressed genes has assisted discovery of gene sets involved in particular biological processes. The purpose of this study was to identify genes involved in appressorium formation in the rice blast fungus Magnaporthe oryzae via analysis of cDNA-amplified fragment length polymorphisms. Amplification of appressorial and vegetative mycelial cDNAs using 28 primer combinations generated over 200 differentially expressed transcript-derived fragments (TDFs). TDFs were excised from gels, re-amplified by PCR, cloned, and sequenced. Forty-four of 52 clones analyzed corresponded to 42 genes. Quantitative real-time PCR showed that expression of 23 genes was up-regulated during appressorium formation, one of which was the $M C K 1$ gene that had been shown to be involved in appressorium formation. This study will be providing valuable resources for identifying the genes such as pathogenicity-related genes in M. oryzae.
\end{abstract}

Keywords: Appressorium formation, cDNA-AFLP, Gene expression, Magnaporthe oryzae, qRT-PCR
Rice blast, caused by Magnaporthe oryzae, is the most devastating disease in rice worldwide (Ebbole, 2007) and destroys $10-30 \%$ of rice production annually (Talbot, 2003). As over half of the global human population depends on rice as staple food (Strange and Scott, 2005), intensive research has been conducted to understand its mechanism of pathogenicity towards the goal of developing effective control strategies for the rice blast.

The infection of rice by $M$. oryzae is initiated by conidial attachment on host surfaces followed by conidial germination. Via recognition of physical cues on the rice surface, such as hydrophobicity and surface hardness, emerging germ tubes initiate formation of a specialized infection structure, called the appressorium, within 4-6 hours (Hamer and Talbot, 1998; Howard and Valent, 1996). The appressorium is a dome-shaped cell that enables the fungus to penetrate the

Research in Plant Disease

pISSN 1598-2262, elSSN 2233-9191

www.online-rpd.org rice leaf surface. Enormous turgor pressure ( $8 \mathrm{MPa}$ ) builds up inside the melanized appressorium due to high glycerol concentration, which drives penetration of the plant surface. After successful infection of plant tissues, visible symptoms appear rapidly. Over two decades of research has unequivocally established the involvement of several signaling pathways, including the cyclic AMP-dependent (Adachi and Hamer, 1998; Lee and Dean, 1993; Mitchell and Dean, 1995; Xu and Hamer, 1996), calcium-dependent (Choi et al., 2009a, 2009b; Liu and Kolattukudy, 1999; Nguyen et al., 2008), and mitogen-activated protein (MAP) kinase signaling pathways (Hamer and Talbot, 1998; Jeon et al., 2008; Soanes et al., 2012; Thines et al., 2000; Xu and Hamer, 1996) in appressorium differentiation.

One notable advantage that has facilitated studies on appressorium differentiation is the possibility to induce appressorium formation on artificial hydrophobic surface as well as hydrophilic surfaces in the presence of CAMP (Lee and Dean, 1993). Multiple approaches have been deployed to identify genes important in M. oryzae appressorium devel- 
opment, including suppression subtractive hybridization (Lu et al., 2005), super serial analysis of gene expression (Irie et al., 2003; Soanes et al., 2012), expressed sequence tag analysis (Oh et al., 2008), and RNA-sequencing (Soanes et al., 2012). Identification of genes expressed in appressoria via such approaches and subsequent functional analysis of identified genes via mutagenesis have helped understand the molecular mechanism underpinning the initiation and maturation of $M$. oryzae appressorium. Even though a large number of genes involved in appressorium differentiation have been identified via functional validation, many more genes likely remain to be identified.

In this study, we applied cDNA-amplified fragment length polymorphisms (CDNA-AFLP) analysis (Park et al., 2009) to identify and characterize the function of selected genes using the previously constructed ATMT mutant library (Jeon et al., 2007).

M. oryzae strain KJ201 (wild-type) was obtained from the Centre for Fungal Genetic Resources at Seoul National University, Seoul, Korea. All fungal isolates were grown on V8juice agar ( $8 \%$ V8 juice, $\mathrm{pH} 6.8$ ) or oatmeal agar (5\% oatmeal $[\mathrm{w} / \mathrm{v}], 2 \%$ agar $[\mathrm{w} / \mathrm{v}])$ at $25^{\circ} \mathrm{C}$ under constant light to promote conidial production. The wild-type and ATMT mutants were cultured on complete agar medium, minimal agar medium, $\mathrm{C}$ starvation medium, and $\mathrm{N}$ starvation medium to observe mycelial growth and colony characteristics (Talbot et al., 1997).

Total RNA was isolated from appressoria, formed on hydrophobic glass for 4-6 hr, and mycelia cultured in complete liquid medium. Extracted RNA $(5 \mu \mathrm{g})$ was used to produce random primed CDNA with the Prom-II Reverse Transcription System (Promega, Madison, WI, USA). Three independent experiments were performed.

This analysis was performed as previously described (Park et al., 2009). cDNA (200 ng) was digested with EcoRl and Mspl and ligated to EcoRl and Mspl double strand adapters (Park et al., 2009). The products were diluted (50X) with TE buffer and $5 \mu$ l were used for selective amplification, using 36 cycles that include 13 touchdown cycles comprising a reduction of the annealing temperature from $65^{\circ} \mathrm{C}$ to $56^{\circ} \mathrm{C}$ in $0.7^{\circ} \mathrm{C}$ steps, which were subsequently maintained for 23 cycles. A total of 28 primer combinations (Table 1) were used for selective amplification as previously described (Park et al., 2009).

Bands of interest were selected, excised from the gel, and re-amplified as previously described (Park et al., 2009). PCR
Table 1. Primers used for CDNA-AFLP

\begin{tabular}{ll}
\hline \multicolumn{1}{c}{ Selective primer } & \multicolumn{1}{c}{$\mathbf{5}^{\prime}$-3' } \\
\hline EcoRl-0 & GAC TGC GTA CCA ATT C \\
EcoRl-AGG & GAC TGC GTA CCA ATT CAG G \\
EcoRI-CAC & GAC TGC GTA CCA ATT CCA C \\
EcoRl-CAG & GAC TGC GTA CCA ATT CCA G \\
Mspl-0 & GAT GAG TCC TGA GCG G \\
Mspl-C & GAT GAG TCC TGA GCG GC \\
Mspl-CT & GAT GAG TCC TGA GCG GCT \\
Mspl-CTC & GAT GAG TCC TGA GCG GCTC \\
Mspl-CTCA & GAT GAG TCC TGA GCG GCT CA \\
Mspl-CTG & GAT GAG TCC TGA GCG GCT G \\
Mspl-CTGC & GAT GAG TCC TGA GCG GCT GC \\
\hline
\end{tabular}

CDNA-AFLP, cDNA-amplified fragment length polymorphisms.

products were confirmed on $1.0 \%$ agarose gels. The amplified transcript-derived fragments (TDFs) were cloned into the plasmid pGEM-T Easy vector system I (Promega). Sequencing of cloned TDFs was conducted on an Applied Biosystems sequencer (ABI3700, Applied Biosystems, Foster City, CA, USA).

Database searches were performed using the BLAST Network Service (NCBI, National Center for Biotechnology Information) and the Magnaporthe genome database (http:// www.broad.mit.edu/annotation/fungi/magnaporthe/). To determine whether the genes derived from TDFs were orphan genes or had orthologs in other organisms, the BLAST Matrix program incorporated in CFGP (Park et al., 2008) and InParanoid algorithms (Remm et al., 2001) were used. We applied the cutoff e-value of less than $10^{-50}$ for protein similarity in BLAST Matrix searches and the InParanoid default parameters.

Quantitative real-time RT-PCR (qRT-PCR) was applied to measure transcript levels using specific primers (Table 2) and the conditions described previously (Park et al., 2013). All reactions were performed with three biological and three technical replicates for each sample. The $\beta$-tubulin gene was used as the internal control for normalization. All amplification curves were analyzed with a normalized reporter threshold of 0.1 to obtain the threshold cycle $(\mathrm{Ct})$ values. The comparative $\Delta \Delta \mathrm{Ct}$ method was applied to evaluate relative quantities of each amplified sample product. Fold changes were 
calculated as $2^{-\Delta \Delta C t}$ (Livak and Schmittgen, 2001). We applied a fold-change cutoff of $\geq 1.5$ for up-regulation and $\leq 0.5$ for down-regulation.

Isolation of differentially expressed TDFs. To isolate TDFs corresponding to differentially expressed genes during appressorium development in $M$. oryzae, cDNA-AFLP analysis was performed using RNAs extracted from appressoriumforming conidia on hydrophobic glass and vegetatively grown mycelia in complete liquid medium.

A total of 28 primer combinations were used (Table 1). Resulting fragments were ranged in size from 50 to $1,200 \mathrm{bp}$. Over 200 TDFs that appeared to be differentially expressed genes were detected. Each band was directly excised from the gel, eluted, and re-amplified using the same primer set. Among the 200 TDFs, 52 were successfully cloned and sequenced. In order to obtain more up-regulated or differentially expressed genes during appressorium development, additional primer combinations for cDNAAFLP could be helpful.

\section{Identification of up-regulated genes during appres-} sorium formation. The GenBank nucleotide sequence database (http://blast.ncbi.nlm.nih.gov/Blast) and Magnaporthe database (http://www.broad.mit.edu/annotation/ fungi/magnaporthe/) were queried using sequences of the 52 TDFs via the BLAST (Basic Local Alignment Search Tool) program. The majority (44 TDFs) matched to M. oryzae genes (Table 3). Based on blast search, we identified 42 independent genes that are likely associated with appressorium development. Among 44 TDFs, 2 TDFs are identical sequence to two TDFs which identified as MGG_01117T0 and MGG_15150T0, respectively.

The genes up-regulated during appressorium development included the following: homologues of the dynactin Arp1 P62 subunit RO2 (MGG_00222T0) (Lee et al., 2001), which regulates the activity of cytoplasmic dynein (a microtubule-associated motor); a gene encoding mitogen activated protein kinase kinase kinase3, MCK1 (MGG_00883T0) (Jeon et al., 2008), which is an integral component of the MAP kinase signaling pathway that regulates cell wall integrity in Saccharomyces cerevisiae (Levin, 2005) and M. oryzae (Jeon et al., 2008); a gene homologous to S. cerevisiae Git1 (MGG_00464T0), which encodes a permease involved in glycerophosphoinositol uptake (Patton-Vogt and Henry,
1998); beta-hexosaminidase (MGG_00621T0) homologue; a homologue of glycosylphosphatidylinositol-anchored extracellular cell wall glucanase Crf1 of Aspergillus fumigatus (MGG_10431T0) (Arroyo et al., 2007); and a pp-loop family protein (MGG_17376T0) homologue (Table 3).

The genes specifically expressed during appressorium development also included a gene putatively encoding acetylglutamate synthase (MGG_01507T0), a gene involved in autophagy; ATG6 (MGG_03694T0), which encodes a protein that forms a membrane-associated complex with ATG14p in S. cerevisiae and is critical for M. oryzae pathogenicity; protein phosphatase 1K (MGG_03918T0); L-ornithine 5-monooxygenase OMO1 (MGG_04212T0); cation efflux family protein (MGG_04407T0); potassiumactivated aldehyde dehydrogenase (MGG_05814T0); nuclear cap-binding protein subunit2 (MGG_06296T0); transport protein SEC23 (MGG_06910T0); CCR4-NOT transcription complex (MGG_08101T0); XPG N-terminal domain-containing protein (MGG_08303T0); cystathionine gamma-lyase (MGG_10380T0); ceramide glucosyltransferase (MGG_10668T0); and DUF124 domain protein (MGG_11494) (Table 3).

Among the 42 genes, only seven (MGG_00222T0, MGG_00464T0, MGG_03694T0, MGG_04538T0, MGG_10380T0, MGG_11809T0, and MGG_15282T0) had already been identified in a previous study (Soanes et al., 2012). The remaining 35 genes were not previously captured in appressorial transcriptome studies in M. oryzae (Oh et al., 2008; Soanes et al., 2012). This low redundancy level compare to other previous transcriptome analysis (Oh et al., 2008; Soanes et al., 2012) might be due to one or more of the following differences among these experiments: (1) differences in experimental methods; (2) inductive surface and treatments for appressorium development; (3) time points for sample collection; (4) differences between strains used; and (5) Magnaporthe genome versions used.

To determine whether the 42 genes have orthologs in other species, we applied BLAST Matrix (http://cfgp.snu. ac.kr) and InParanoid (Remm et al., 2001) to detect orthologs (Fig. 1). We found 5 genes (MGG_14593T0, MGG_15150 T0, MGG_14683 T0, MGG_14684 T0, and MGG_14741 T0) were M. oryzae-specific, based on the absence of orthologs in other species (BLAST E $<10^{-50}$ and InParanoid) (Fig. 1). Further examination is needed to determine whether these genes play an important role during $M$. oryzae appressorium devel- 
Table 2. Primers used for quantitative real-time PCR

\begin{tabular}{|c|c|c|}
\hline Gene locus & Forward primer $\left(5^{\prime}-3^{\prime}\right)$ & Reverse primer $\left(5^{\prime}-3^{\prime}\right)$ \\
\hline MGG_00204T0 & GTCAAACATTTACCTCGCGC & CATAAAGATTGAACCGCCCAG \\
\hline MGG_00222T0 & САCCATCTACTCAAACCCCTC & TCGAATTGTGGACAGAGCAAG \\
\hline MGG_00464T0 & TCCTCTTCACCAACAGCATG & GACCAGAGGAAATACGACACC \\
\hline MGG_00621T0 & TGTGACCTTGTGCTCTTGTG & GACGTGCAACCTCTITICATG \\
\hline MGG_00752T0 & CTITGTCGGTGTITTCCTCAG & GCAGCGGAACGAATTGAAG \\
\hline MCK1 & GGTITIATGCCGGATTTGAGC & TCTGTGGGAGCATTTGACTG \\
\hline MGG_01117T0 & TCCCAGAAAGTGACCATCAAG & GGTCCAAGAACTGAGTGAAGG \\
\hline MGG_01507T0 & ATGCCGTCAGAAGCTTATCG & CTGCCATTTCTTATCTCTITCCG \\
\hline MGG_02313T0 & ACAGTATTTCTCCGCCTCAAG & TGCCAATATTCGTCTGTGTCC \\
\hline MGG_03178T0 & GCCAAGATCATACACTAGACGAG & AATCTAACTTGGCTGTCGGG \\
\hline MGG_03531T0 & TGGCTTTGATACTGGAACGAG & TGAGTGGTTCATGAGGGTTG \\
\hline MGG_03694T0 & CCACGATCAGAACAGGAAGG & CAGGATAAAGGACATGGAGCTG \\
\hline MGG_03918T0 & TCAGCGAATACAAGGACACG & СTCTTGGTGATACTGAAGTGGG \\
\hline MGG_04121T0 & TTGTCTCGTATGGCTTCAGTG & TTCTTAAGTCTCGCGCCAAG \\
\hline MGG_04212T0 & CAATGGCACAAACGGTACAG & CAAACCCCACGCAAATCAG \\
\hline MGG_04407T0 & GTCTGTTGAGAAGCTGTTGC & GGACTAGAGCTTGAACTTGGG \\
\hline MGG_04538T0 & AGATGTGCCATATTTGCCCC & TGACTGTAGCGTTCTCCAAAG \\
\hline MGG_05592T0 & AGCTCATCGACTTTGCCTAC & GGGTTCGGTCTCGTAGAAG \\
\hline MGG_05814T0 & ATCTTGGTCAACTCGCTCATC & AGTGAAAGTACTGGATGACGC \\
\hline MGG_06296T0 & CCAACTCGACAACGCTCTATG & ACGAAGCAGAAACCGCAG \\
\hline MGG_06910T0 & TGCCAGGTTGATGTTCGTG & CCGTGATGTCCTTGTAGTGAG \\
\hline MGG_06999T0 & TCCAAAAGTCTCAGTGTCGC & AATCTGCCCCTGAAACGG \\
\hline MGG_08101T0 & CAAATGGAGAGGTTCAAAGCG & TGTCGATCATGTTCCCAAGG \\
\hline MGG_08114T0 & AGCCATGTTAAGGAGATGCAG & TСАGTCCATTCATCCCACG \\
\hline MGG_08303T0 & ATGTGGCGATGAAGGTCTG & GATTTGAGTTGGCTGTCCGTG \\
\hline MGG_10035T0 & CATAGTCATTCTTGTCCCACCC & ATGAGCTTGTTCTCGGTCG \\
\hline MGG_10380T0 & TGACCTITAGCCACGACATTG & TCAATCCAGATGAGCTTGGTG \\
\hline MGG_10431T0 & AAACCAAACAATGCAGCCG & CCCCATGTACTTGTAGCAGTAG \\
\hline MGG_10668T0 & ACTACCTAAAGAAACGGCGAC & CCAGATCGCCAAATACTAGACC \\
\hline MGG_11494T0 & AGTGGACAACGGGCATTAG & GCAACACCAGACACTATACCTC \\
\hline MGG_11809T0 & CATCACACTTGGCTITIGGG & TCCCTTCATTGTCCCCTTTG \\
\hline MGG_13902T0 & TCATCGACCTITTCAGCCG & AAACAAGTCTCCCAGGTTCC \\
\hline MGG_14593T0 & GAGGCCGAATGTTIAGAGCTAG & GGCATGACTITGTTITCGTTG \\
\hline MGG_14683T0 & GCACTTTCACATCTTTCTCGG & TTGAGCGTCTGAGTTGAGG \\
\hline MGG_14684T0 & GTGATCAGCTCGTACCTCAG & ATCTGCGCTGTATCCCATG \\
\hline MGG_14741T0 & AGCAGGTAAGGCCAACTAATG & GAGTACTTGGACATCGGAATCG \\
\hline MGG_15150T0 & TCACGAAAACCCCATCTCATG & TGATGTTGAAGGAGCGAGTG \\
\hline MGG_15282T0 & TTCGAGTCATTGGGATCTTGG & AGGAACTTGGTCGGCATATC \\
\hline MGG_16167T0 & CTTGCCATCAAACTCGACAAC & TाCTGTGGTATGTGGGTTGG \\
\hline MGG_16535T0 & CAGATAGAGCAACAGAGGCAG & GTCCATGGTCCTGCATTITG \\
\hline MGG_17376T0 & GCCGATGCTTGTATTACCAAC & CAGAGTATGAGGTGCTGTAGTG \\
\hline MGG_17847T0 & CTTGCCATCAAACTCGACAAC & TTCTGTGGTATGTGGGTTGG \\
\hline$\beta$-Tubulin & ACAACTTCGTCTTCGGTCAG & GTGATCTGGAAACCCTGGAG \\
\hline
\end{tabular}


Table 3. List of 44 transcript-derived fragments corresponding to 42 genes and the relative abundance of their transcripts during appressorium development

\begin{tabular}{|c|c|c|}
\hline Locus & a.a. length & Putative function \\
\hline MGG_00204T0 & 515 & Hypothetical protein \\
\hline MGG_00222T0 & 652 & Dynactin Arp1 p62 subunit RO2 \\
\hline MGG_00464T0 & 516 & MFS phospholipid transporter Git1 \\
\hline MGG_00621T0 & 948 & Beta-hexosaminidase \\
\hline MGG_00752T0 & 197 & Hypothetical protein \\
\hline MGG_00883T0 & 1,528 & Mitogen activated protein kinase kinase kinase 3 \\
\hline MGG_01117T0 & 740 & Conserved hypothetical protein \\
\hline MGG_01507T0 & 694 & Acetylglutamate synthase \\
\hline MGG_02313T0 & 3,298 & Conserved hypothetical protein \\
\hline MGG_03178T0 & 229 & Conserved hypothetical protein \\
\hline MGG_03531T0 & 571 & Conserved hypothetical protein \\
\hline MGG_03694T0 & 503 & Beclin-1 \\
\hline MGG_03918T0 & 696 & Protein phosphatase $1 \mathrm{~K}$, mitochondrial precursor \\
\hline MGG_04121T0 & 249 & Hypothetical protein \\
\hline MGG_04212T0 & 563 & L-ornithine 5-monooxygenase (OMO1) \\
\hline MGG_04407T0 & 481 & Cation efflux family protein \\
\hline MGG_04538T0 & 584 & Conserved hypothetical protein \\
\hline MGG_05592T0 & 541 & Conserved hypothetical protein \\
\hline MGG_05814T0 & 470 & Potassium-activated aldehyde dehydrogenase \\
\hline MGG_06296T0 & 185 & Nuclear cap-binding protein subunit 2 \\
\hline MGG_06910T0 & 770 & Transport protein SEC23 \\
\hline MGG_06999T0 & 514 & Hypothetical protein \\
\hline MGG_08101T0 & 665 & CCR4-NOT transcription complex \\
\hline MGG_08114T0 & 855 & Hypothetical protein \\
\hline MGG_08303T0 & 734 & XPG N-terminal domain-containing protein \\
\hline MGG_10035T0 & 299 & Conserved hypothetical protein \\
\hline MGG_10380T0 & 426 & Cystathionine gamma-lyase \\
\hline MGG_10431T0 & 793 & Extracellular cell wall glucanase Crf1 \\
\hline MGG_10668T0 & 494 & Ceramide glucosyltransferase \\
\hline MGG_11494T0 & 478 & DUF124 domain protein \\
\hline MGG_11809T0 & 740 & Hypothetical protein \\
\hline MGG_13902T0 & 802 & Conserved hypothetical protein \\
\hline MGG_14593T0 & 312 & Hypothetical protein \\
\hline MGG_14683T0 & 594 & Hypothetical protein \\
\hline MGG_14684T0 & 60 & Hypothetical protein \\
\hline MGG_14741T0 & 245 & Predicted protein \\
\hline MGG_15150T0 & 108 & Conserved hypothetical protein \\
\hline MGG_15282T0 & 619 & Conserved hypothetical protein \\
\hline MGG_16167T0 & 535 & Hypothetical protein \\
\hline MGG_16535T0 & 224 & Hypothetical protein \\
\hline MGG_17376T0 & 83 & PP-loop family protein \\
\hline MGG_17847T0 & 728 & Hypothetical protein \\
\hline
\end{tabular}




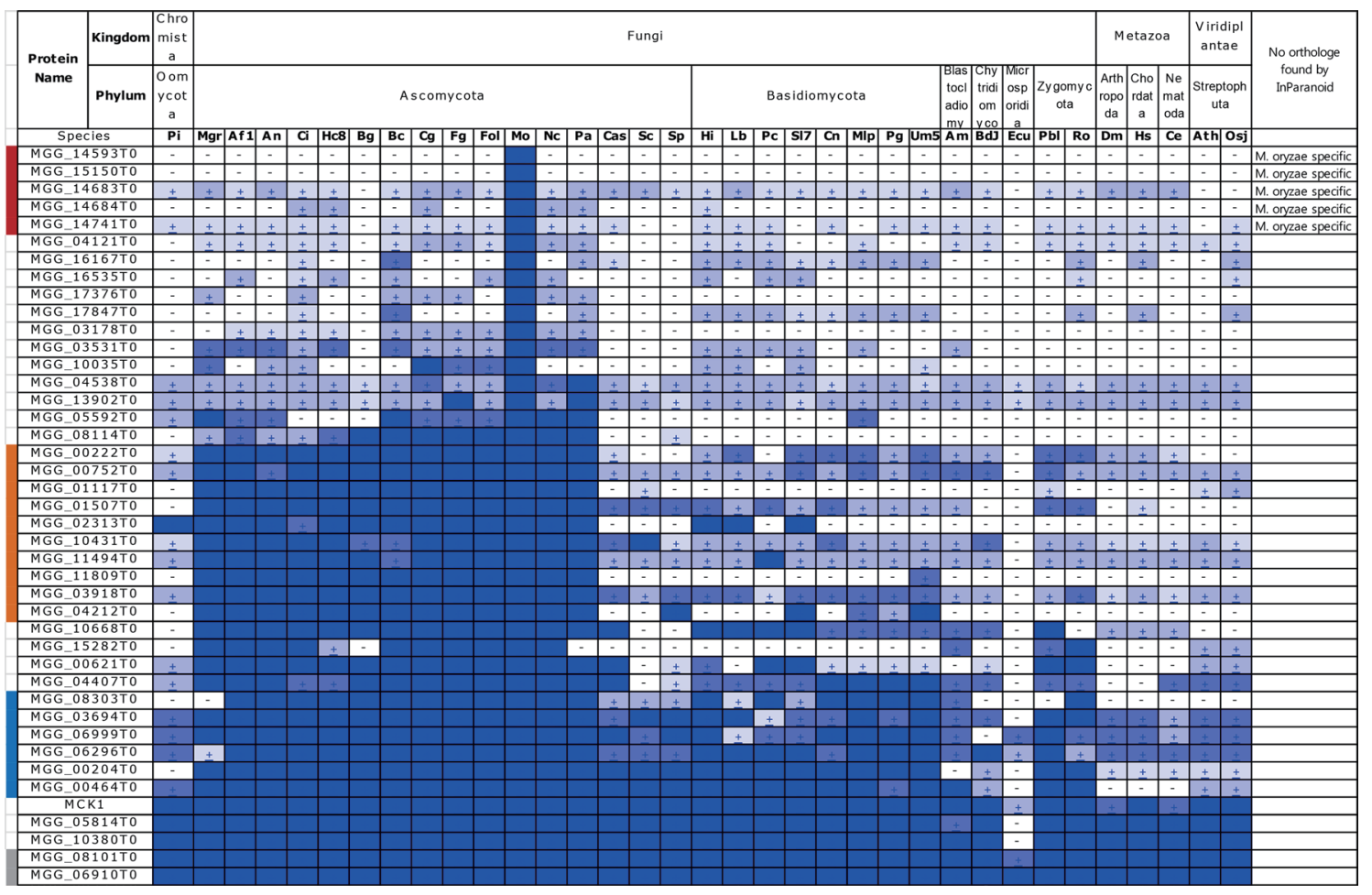

Fig. 1. Distribution of 42 appressorium-specific genes analyzed using BLAST Matrix and InParanoid algorism. Abbreviation for fungal species and other organisms: Pi, Phytophthora infestans; Mgr, Mycosphaerella graminicola; Af1, Aspergillus fumigatus Af293; An, Aspergillus nidulans; $\mathrm{Ci}$, Coccidioides immitis; $\mathrm{Hc} 8$, Histoplasma capsulatum H88; Bg, Blumeria graminis f. sp. hordei; $\mathrm{Bc}$, Botrytis cinerea; Cg, Colletotrichum graminicola M1.001; Fg, Fusarium graminearum; Fol, Fusarium oxysporum; Mo, Magnaporthe oryzae 70-15; Nc, Neurospora crassa; Pa, Podospora anserina; Cas, Candida albicans SC5314; Sc, Saccharomyces cerevisiae S288C; Sp, Schizosaccharomyces pombe; Hi, Heterobasidion irregulare TC32-1; Lb, Laccaria bicolor; Pc, Phanerochaete chrysosporium; SI7, Serpula lacrymans S7.3; Ecu, Encephalitozoon cuniculi JAM81; Pbl, Phycomyces blakesleeanus; Ro, Rhizopus oryzae; Dm, Dorosophila melanogaster; Hs, Homo sapiens; Ce, Caenorhabditis elegans; Ath, Arabidopsis thaliana; Osj, Oryza sativa japonica. Red bar, M. oryzae-specific; orange bar, Ascomycota-specific; blue bar, fungal-specific; gray bar, conserved in whole organisms that we used in this study.

opment and/or pathogenesis.

We also found that 10 genes (MGG_00222T0, MGG_00752T0, MGG_01117T0, MGG_01507T0, MGG_02313T0, MGG_10431T0, MGG_11494T0, MGG_11809T0, MGG_03918T0, and MGG_04212T0) were Ascomycota-specific (particularly in Pezizomycotina), 6 additional genes (MGG_08303T0, MGG_03694T0, MGG_06999T0, MGG_06296T0, MGG_00204T0, and MGG_00464T0) were fungal-specific, and 2 genes (MGG_08101T0 and MGG_06910T0) were conserved in all organisms included in the analysis (Fig. 1).

Validation of expression patterns by qRT-PCR. To validate gene expression patterns suggested by cDNA-AFLP analysis, qRT-PCR analysis was conducted. A total of 23 genes (54.8\%) were up-regulated, 9 genes (21.4\%) were down- regulated, and 10 genes (23.8\%) were not differentially expressed (Fig. 2).

MGG_04212T0 encodes an L-ornithine 5-monooxygenase (OMO1), which is the key precursor for all hydroxamate siderophore biosynthesis in fungi (Eisendle et al., 2003; Schrettl et al., 2007). The siderophore is known to be required for virulence in A. fumigatus (Schrettl et al., 2007). We found that OMO1 expression was significantly up-regulated during appressorium formation (Fig. 1). Previous studies revealed that overcoming host-driven oxidative stress was a prerequisite for full pathogenicity in rice blast fungus (Chi et al., 2009; Huang et al., 2011). We previously showed homeostatic regulation of ferrous ion might serve an important role in pathogenicity via overcoming oxidative stress (Chi et al., 2009).

Similarly, MGG_04407T0, which encodes a putative cation efflux protein, and is also required for regulation of metal ion 


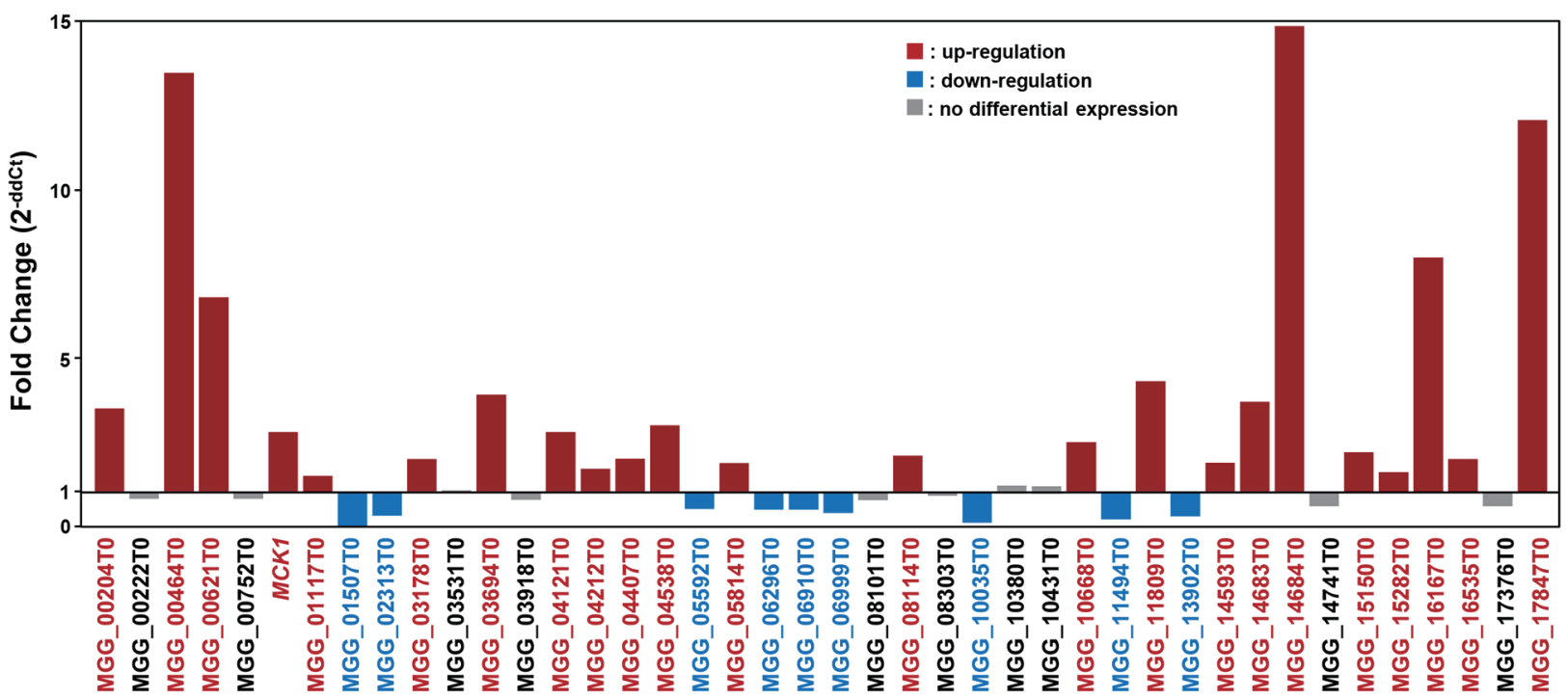

Fig. 2. Quantitative real-time PCR analysis of gene expression during appressorium development. Up-regulated genes (more than 2 fold) are indicated by red bars, down-regulated genes (less than 0.5 fold) are marked by blue bars. The genes did not show differentially expression are noted in black.

concentration. Further investigation is required to elucidate the roles of MGG_04212T0 and MGG_04407T0 in M. oryzae pathogenicity and metal ion regulation.

CDNA-AFLP, sequencing and qRT-PCR can be combined for effective identification of differentially expressed genes under specific conditions. Using these approaches, we have identified and confirmed differentially expressed genes during appressorium formation in $M$. oryzae. Subsequent expression, bioinformatics, and functional validation revealed interesting pathogenicity-related genes in $M$. oryzae. Further gene knock-out analysis would determine the role of these genes in appressorium development and/ or pathogenicity.

\section{Conflicts of Interest}

No potential conflict of interest relevant to this article was reported.

\section{Acknowledgments}

The authors grateful to Jongbum Jeon for assistance in analysis of overall data. This work was supported by the National Research Foundation of Korea grant funded by the Korean government (NRF-2017R1D1A1B04035888).

\section{References}

Adachi, K. and Hamer, J. E. 1998. Divergent cAMP signaling pathways regulate growth and pathogenesis in the rice blast fungus Magnaporthe grisea. Plant Cell 10: 1361-1374.

Arroyo, J., Sarfati, J., Baixench, M.T., Ragni, E., Guillén, M., RodriguezPeña, J. M. et al. 2007. The GPI-anchored gas and Crh families are fungal antigens. Yeast 24: 289-296.

Chi, M.-H., Park, S.-Y., Kim, S. and Lee, Y.-H. 2009. A novel pathogenicity gene is required in the rice blast fungus to suppress the basal defenses of the host. PLoS Pathog. 5: e1000401.

Choi, J., Kim, Y., Kim, S., Park, J. and Lee, Y.-H. 2009a. MoCRZ1, a gene encoding a calcineurin-responsive transcription factor, regulates fungal growth and pathogenicity of Magnaporthe oryzae. Fungal Genet. Biol. 46: 243-254.

Choi, J. H., Kim, Y. and Lee, Y.-H. 2009b. Functional analysis of MCNA, a gene encoding a catalytic subunit of calcineurin, in the rice blast fungus Magnaporthe oryzae. J. Microbiol. Biotechnol. 19: 11-16.

Ebbole, D. J. 2007. Magnaporthe as a model for understanding hostpathogen interactions. Annu. Rev. Phytopathol. 45: 437-456.

Eisendle, M., Oberegger, H., Zadra, I. and Haas, H. 2003. The siderophore system is essential for viability of Aspergillus nidulans: functional analysis of two genes encoding l-ornithine N 5-monooxygenase (sidA) and a non-ribosomal peptide synthetase (sidC). Mol. Microbiol. 49: 359-375.

Hamer, J. E. and Talbot, N. J. 1998. Infection-related development in the rice blast fungus Magnaporthe grisea. Curr. Opin. Microbiol. 1: 693-697.

Howard, R. J. and Valent, B. 1996. Breaking and entering: host pen- 
etration by the fungal rice blast pathogen Magnaporthe grisea. Annu. Rev. Microbiol. 50: 491-512.

Huang, K., Czymmek, K. J., Caplan, J. L., Sweigard, J. A. and Donofrio, N. M. 2011. HYR1-mediated detoxification of reactive oxygen species is required for full virulence in the rice blast fungus. PLoS Pathog. 7: e1001335.

Irie, T., Matsumura, H., Terauchi, R. and Saitoh, H. 2003. Serial Analysis of Gene Expression (SAGE) of Magnaporthe grisea: genes involved in appressorium formation. Mol. Genet. Genomics 270: 181-189.

Jeon, J., Goh, J., Yoo, S., Chi, M.-H., Choi, J., Rho, H.-S. et al. 2008. A putative MAP kinase kinase kinase, $M C K 1$, is required for cell wall integrity and pathogenicity of the rice blast fungus, Magnaporthe oryzae. Mol. Plant Microbe Interact. 21: 525-534.

Jeon, J., Park, S.-Y., Chi, M.-H., Choi, J., Park, J., Rho, H.-S. et al. 2007. Genome-wide functional analysis of pathogenicity genes in the rice blast fungus. Nat. Genet. 39: 561-565.

Lee, I. H., Kumar, S. and Plamann, M. 2001. Null mutants of the neurospora actin-related protein 1 pointed-end complex show distinct phenotypes. Mol. Biol. Cell 12: 2195-2206.

Lee, Y.-H. and Dean, R. A. 1993. cAMP regulates infection structure formation in the plant pathogenic fungus Magnaporthe grisea. Plant Cell 5: 693-700.

Levin, D. E. 2005. Cell wall integrity signaling in Saccharomyces cerevisiae. Microbiol. Mol. Biol. Rev. 69: 262-291.

Liu, Z.-M. and Kolattukudy, P. E. 1999. Early expression of the calmodulin gene, which precedes appressorium formation in Magnaporthe grisea, is inhibited by self-inhibitors and requires surface attachment. J. Bacteriol. 181: 3571-3577.

Livak, K. J. and Schmittgen, T. D. 2001. Analysis of relative gene expression data using real-time quantitative PCR and the 2(-Delta Delta C(T)) method. Methods 25: 402-408.

Lu, J.-P., Liu, T.-B. and Lin, F.-C. 2005. Identification of mature appressorium-enriched transcripts in Magnaporthe grisea, the rice blast fungus, using suppression subtractive hybridization. FEMS Microbiol. Lett. 245: 131-137.

Mitchell, T. K. and Dean, R. A. 1995. The cAMP-dependent protein kinase catalytic subunit is required for appressorium formation and pathogenesis by the rice blast pathogen Magnaporthe grisea. Plant Cell 7: 1869-1878.

Nguyen, Q. B., Kadotani, N., Kasahara, S., Tosa, Y., Mayama, S. and Nakayashiki, H. 2008. Systematic functional analysis of calciumsignalling proteins in the genome of the rice-blast fungus, Magnaporthe oryzae, using a high-throughput RNA-silencing system. Mol. Microbiol. 68: 1348-1365.
Oh, Y., Donofrio, N., Pan, H., Coughlan, S., Brown, D. E., Meng, S. et al. A. 2008. Transcriptome analysis reveals new insight into appressorium formation and function in the rice blast fungus Magnaporthe oryzae. Genome Biol. 9: R85.

Park, J., Park, B., Jung, K., Jang, S., Yu, K., Choi, J. et al. 2008. CFGP: a web-based, comparative fungal genomics platform. Nucleic Acids Res. 36: D562-D571.

Park, S.-Y., Choi, J., Lim, S.-E., Lee, G.-W., Park, J., Kim, Y. et al. 2013. Global expression profiling of transcription factor genes provides new insights into pathogenicity and stress responses in the rice blast fungus. PLoS Pathog. 9: e1003350.

Park, S.-Y., Jwa, N.-S., Chi, M.-H. and Lee, Y.-H. 2009. A fluorescencebased cDNA-AFLP method for identification of differentially expressed genes. Plant Pathol. J. 25: 184-188.

Patton-Vogt, J. L. and Henry, S. A. 1998. GIT1, a gene encoding a novel transporter for glycerophosphoinositol in Saccharomyces cerevisiae. Genetics 149: 1707-1715.

Remm, M., Storm, C. E. V. and Sonnhammer, E. L. L. 2001. Automatic clustering of orthologs and in-paralogs from pairwise species comparisons. J. Mol. Biol. 314: 1041-1052.

Schrettl, M., Bignell, E., Kragl, C., Sabiha, Y., Loss, O., Eisendle, M. et al. 2007. Distinct roles for intra- and extracellular siderophores during Aspergillus fumigatus infection. PLoS Pathog. 3: 1195-1207.

Soanes, D. M., Chakrabarti, A., Paszkiewicz, K. H., Dawe, A. L. and Talbot, N. J. 2012. Genome-wide transcriptional profiling of appressorium development by the rice blast fungus Magnaporthe oryzae. PLoS Pathog. 8: e1002514.

Strange, R. N. and Scott, P. R. 2005. Plant disease: a threat to global food security. Annu. Rev. Phytopathol. 43: 83-116.

Talbot, N. J. 2003. On the trail of a cereal killer: exploring the biology of Magnaporthe grisea. Annu. Rev. Microbiol. 57: 177-202.

Talbot, N. J., Mccafferty, H. R. K., Ma, M., Koore, K. and Hamer, J. E. 1997. Nitrogen starvation of the rice blast fungus Magnaporthe grisea may act as an environmental cue for disease symptom expression. Physiol. Mol. Plant Pathol. 50: 179-195.

Thines, E., Weber, R. W. S. and Talbot, N. J. 2000. MAP kinase and protein kinase A-dependent mobilization of triacylglycerol and glycogen during appressorium turgor generation by Magnaporthe grisea. Plant Cell 12: 1703-1718.

Xu, J.-R. and Hamer, J. E. 1996. MAP kinase and CAMP signaling regulate infection structure formation and pathogenic growth in the rice blast fungus Magnaporthe grisea. Genes Dev. 10: 26962706. 